\title{
Controlling Electron-Phonon Interactions in Graphene at Ultrahigh Carrier Densities
}

\author{
Dmitri K. Efetov and Philip Kim \\ Department of Physics, Columbia University New York, New York 10027, USA
}

(Received 13 September 2010; published 13 December 2010)

\begin{abstract}
We report on the temperature dependent electron transport in graphene at different carrier densities $n$. Employing an electrolytic gate, we demonstrate that $n$ can be adjusted up to $4 \times 10^{14} \mathrm{~cm}^{-2}$ for both electrons and holes. The measured sample resistivity $\rho$ increases linearly with temperature $T$ in the high temperature limit, indicating that a quasiclassical phonon distribution is responsible for the electron scattering. As $T$ decreases, the resistivity decreases more rapidly following $\rho(T) \sim T^{4}$. This low temperature behavior can be described by a Bloch-Grüneisen model taking into account the quantum distribution of the two-dimensional acoustic phonons in graphene. We map out the density dependence of the characteristic temperature $\Theta_{\mathrm{BG}}$ defining the crossover between the two distinct regimes, and show that, for all $n, \rho(T)$ scales as a universal function of the normalized temperature $T / \Theta_{\mathrm{BG}}$.

At finite temperatures electrons in typical conductors are scattered by phonons, producing a finite, temperature dependent resistivity $\rho$ [1]. If the temperature $T$ is comparable to or larger than the Debye temperature $\Theta_{D}$ - the representative temperature scale for the highest phonon energies-all phonon modes are populated. In this high temperature regime, $\rho(T) \sim T$, reflecting a classical equipartition distribution of the phonons. As $T$ decreases below $\Theta_{D}$, however, the bosonic nature of the phonons becomes important: only the acoustic phonon modes within the phonon sphere of diameter $k_{\mathrm{ph}}=k_{B} T / \hbar v_{s}<k_{D}$ (where $k_{D}$ is the radius of the Debye sphere and $v_{s}$ is the sound velocity) are populated appreciably, leading to a more rapid decrease of the resistivity, $\rho(T) \sim T^{5}$, known as the Bloch-Grüneisen (BG) regime for typical threedimensional (3D) metals [2-4].

Because of the quasielasticity of the electron-phonon $(e-\mathrm{ph})$ interactions, the maximal phonon momentum in an $e$-ph scattering event is limited to $2 \hbar k_{F}$, representing a full backscattering of the electrons across the Fermi surface of radius $k_{F}$. Since in metals $k_{F}$ is of the size of the Brillouin Zone (BZ), $2 k_{F}>k_{D}$, all populated phonons can scatter off electrons. For low density electron systems, however, the Fermi surface can be substantially smaller than the size of the $\mathrm{BZ}$, and hence $k_{F} \ll k_{D}$. In this case, only a small fraction of the acoustic phonons with energies $\hbar v_{s} k_{\mathrm{ph}} \leq 2 \hbar v_{s} k_{F}$ can scatter off electrons. This phase space restriction defines a new characteristic temperature scale for the low density $e$-ph scattering, the BG temperature $\Theta_{\mathrm{BG}}=2 \hbar v_{s} k_{F} / k_{B}<\Theta_{D}$. It was explicitly shown in low density two-dimensional (2D) electron gases formed in semiconductor heterojunctions [5] that $\rho(T)$ drops precipitously at temperatures below $T<\Theta_{\mathrm{BG}}$ rather than $\Theta_{D}$. However, the 3D nature of the phonons in the host material and the low level of tunability of $n$ make this system ineligible for studying 2D BG physics and the explicit density dependence of $\Theta_{\mathrm{BG}}$.
The advance of graphene [6,7] brings new aspects to the study of $e$-ph interactions in a low dimensional system. Graphene has a Debye temperature $\Theta_{D} \approx 2300 \mathrm{~K}$ almost an order of magnitude higher than for typical metallic systems, and the electrostatic tunability of $k_{F} \propto \sqrt{n}$ allows for a wide range of control of $\Theta_{\mathrm{BG}}$. In addition, the single atomic plane structure of graphene provides not only a strictly 2D electronic system, but a 2D acoustic phonon system as well. These unique properties have been considered theoretically, leading to the prediction that graphene exhibits a smooth crossover behavior between the high temperature $\rho(T) \sim T$ and the low temperature $\rho(T) \sim T^{4}$ dependence [8]. The slower reduction of $\rho(T)$ at low $T$ as compared to the $T^{5}$ dependence observed in typical 3D conductors can be understood by the reduced spatial dimensionality.

In this Letter, we report the experimental observation of a 2D BG behavior in graphene. Using an electrolytic gate, we achieve extremely high carrier densities up to $4 \times 10^{14} \mathrm{~cm}^{-2}$ for both electrons and holes, tuning $\Theta_{\mathrm{BG}}$ to values of up to $\sim 1000 \mathrm{~K}$. In the low $T$ limit, $T \ll \Theta_{\mathrm{BG}}$, a $\rho(T) \sim T^{4}$ is observed, reflecting the $2 \mathrm{D}$ nature of the electrons and the acoustic phonons in graphene. At high temperatures, the resistivity shows a semiclassical $\rho(T) \sim T$ behavior. From analysis of the experimental data of $\rho(T)$, we obtain $\Theta_{\mathrm{BG}}(n)$ and show that $\rho(T)$ scales as a universal function $\rho\left(T / \Theta_{\mathrm{BG}}\right)$ of the normalized temperature $T / \Theta_{\mathrm{BG}}$ for all densities $|n|$, which agrees well with the theoretical expressions.

In previous graphene experiments employing thermally grown $\mathrm{SiO}_{2}$ layers as the gate dielectric $[9,10], \rho(T)$ could be measured only in the density range $|n|<5 \times$ $10^{12} \mathrm{~cm}^{-2}$. In this relatively low range of carrier density, $\rho(T) \sim T$ was reported for all $T<150 \mathrm{~K}$, and, at higher temperatures, $T \gtrsim 150 \mathrm{~K}, \rho(T)$ exhibited a rapid increase presumably due to the scattering by thermally activated $\mathrm{SiO}_{2}$ polar optical phonons [10], by thermally quenched 
graphene ripples $[9,11]$, or by Coulomb impurities [12]. In addition, the low temperature behavior of $\rho(T)$ below $T<20 \mathrm{~K}$ was found to be completely dominated by disorder in the samples. These extrinsic effects become less pronounced at higher carrier densities, where the carrier screening is enhanced [10]. Furthermore, an increased carrier density would result in an increase of $\Theta_{\mathrm{BG}}$, allowing us to access the nonlinear $\rho(T)$ of the BG regime in a much wider temperature range.

In order to increase the electrostatic doping, we employ a solid polymer electrolyte gate $[13,14]$. This approach has previously been applied to graphene samples [15-17], and carrier densities of $n \sim 10^{13} \mathrm{~cm}^{-2}$ have been reported under ambient conditions. Following this experimental approach, we increase the efficiency of the electrolyte gate by employing a rapid cooling method which prevents sample degradation, and reach $n>10^{14} \mathrm{~cm}^{-2}$ for both electrons and holes.

Figure 1(a) shows a working principle of the solid polymer electrolyte gate used in our experiment. $\mathrm{Li}^{+}$and $\mathrm{ClO}_{4}^{-}$ions are mobile within a solid "mesh" formed by the polymer poly(ethylene)oxide. By applying a voltage $V_{\text {eg }}$ to
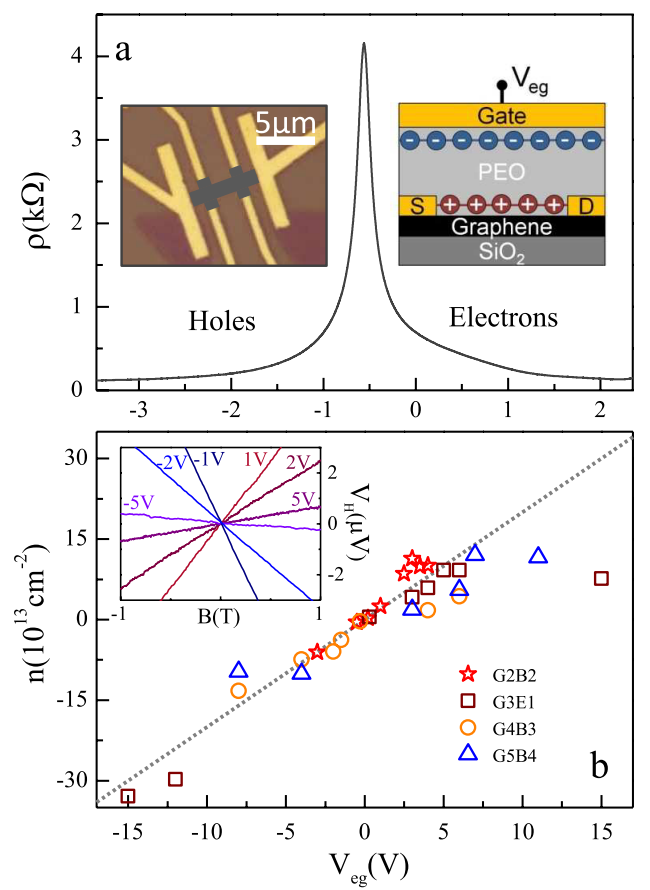

FIG. 1 (color online). (a) Resistivity as a function of applied electrolyte gate voltage $V_{\text {eg }}$ at $T=300 \mathrm{~K}$. Right inset: a schematic view of the electrolyte gated device. The Debye layers are formed $d \sim 1 \mathrm{~nm}$ above the graphene surface. The left inset shows an optical microscope image (false colors) of a typical etched Hall bar device. (b) The inset shows the Hall voltage $V_{H}$ as a function of the magnetic field $B$ for different $V_{\text {eg }}$ (current across the sample $I=100 \mathrm{nA}$ ). The main panel shows the extracted densities $n$ from the various Hall measurements as a function of $V_{\text {eg }}$ for four different samples. The slope of the line fit represents the capacitive coupling of the electrolyte gate to the graphene. the electrolyte gate, the ions form Debye layers on top of the graphene and the gate electrode, respectively. The extreme proximity of these charged layers, separated only by the Debye length $\lambda_{D} \sim 1 \mathrm{~nm}$ from the graphene surface, results in huge capacitances per unit area $C_{\mathrm{eg}}=$ $1 / \epsilon \lambda_{D}$. Under ambient conditions the electrolyte gate $V_{\mathrm{eg}}$ can be swept continuously, doping the graphene samples to either electrons or holes, inducing a modulation of $\rho\left(V_{\mathrm{eg}}\right)$, Fig. 1(a).

The maximal $|n|$ that can be induced by the solid polymer electrolyte is mainly limited by the onset of electrochemical reactions of the ions with the graphene, which typically turn on when $V_{\mathrm{eg}} \geq 3 \mathrm{~V}$ are applied. Although the threshold of the electrochemistry, signaled by a steady increase of $\rho$ with time, depends on the details of the particular device and sample quality, the time span until complete degradation of the sample is typically just a few minutes. We could apply extremely high $V_{\text {eg }}$ of up to $15 \mathrm{~V}$ to the electrolyte, avoiding electrochemically induced sample degradation by immediate cooling of the sample $(<1 \mathrm{~min}$ ) below $T<250 \mathrm{~K}$. At this temperature, both the $\mathrm{Li}^{+}$and $\mathrm{ClO}_{4}^{-}$ions "freeze" out and are no longer mobile within the poly(ethylene)oxide, fixing the accumulated charges in the Debye layers to the graphene surface. The induced charge carrier densities do not vary significantly over time and temperature until after the sample is warmed up again. Accumulated $|n|$ for each applied gate voltage $V_{\text {eg }}$ are characterized directly by performing Hall measurements [Fig. 1(b) inset). Figure 1(b) shows the measured $n$ as a function of $V_{\mathrm{eg}}$. The capacitive coupling of the electrolyte can then be estimated from the slope of $n\left(V_{\mathrm{eg}}\right)$, where we obtain $C_{\mathrm{eg}} \approx 3.2 \mu \mathrm{F} \mathrm{cm}^{-2}$, which is more than 250 times higher than that for typical $300 \mathrm{~nm}$ thick $\mathrm{SiO}_{2} / \mathrm{Si}$ back gates.

In this experiment we have measured $\rho(T)$ for more than 10 single layer graphene samples, in the temperature range $1.5<T<300 \mathrm{~K}$ and for $|n|<2 \times 10^{14} \mathrm{~cm}^{-2}$. Figure 2(a) shows a representative data set for the measured $\rho(T)$ at various fixed $n$. Generally, $\rho(T)$ decreases monotonically as $T$ decreases, saturating to $\rho_{0}$ in the low temperature limit [18]. This residual resistance $\rho_{0}$ stems from the electron scattering on static impurities and point defects and is expected to be almost temperature independent as was discussed in previous studies $[9,10]$. As shown in the inset of Fig. 2(b), the corresponding mobility follows $\mu_{0}^{-1}=$ $\left(\rho_{0} e n\right) \approx a+b n$ [19], with the fitting parameters $a=$ $3.3 \times 10^{-4} \mathrm{~V} \mathrm{~s} / \mathrm{cm}^{2}$ and $b=3.9 \times 10^{-18} \mathrm{~V} \mathrm{~s}$ representing long and short range impurity scattering, respectively.

At first glance, the temperature dependent $\rho(T)$ can be subdivided into two different temperature regimes: (i) the high temperature linear $T$ regime [20] and (ii) the low temperature nonlinear $T$ regime. This transitional trend of $\rho(T)$ at low temperatures can be better scrutinized by subtracting $\rho_{0}$ from $\rho(T)$. Figure 2(b) displays $\Delta \rho(T)=$ $\rho(T)-\rho_{0}$ as a function of $T$ in the logarithmic scale. 

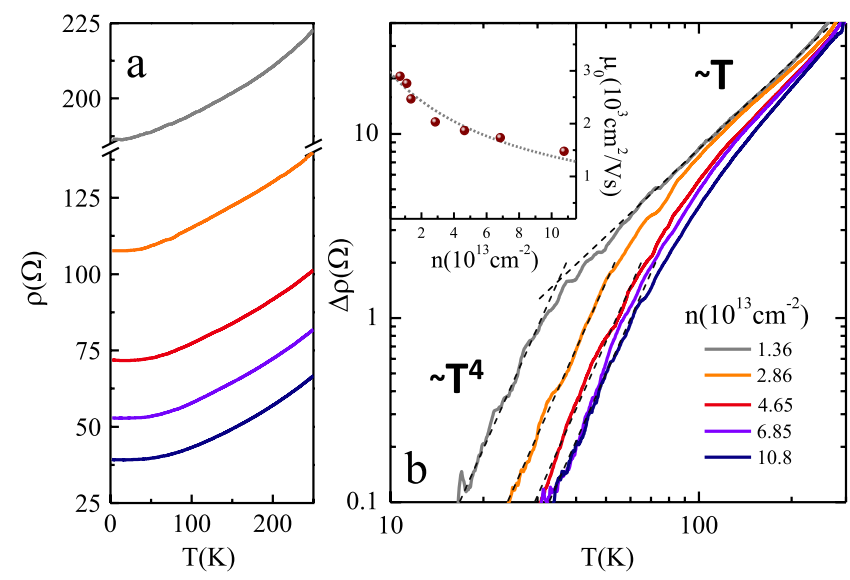

FIG. 2 (color online). (a) Temperature dependence of the resistivity for different charge carrier densities of sample G8A4. (b) The temperature dependent part of the resistivity $\Delta \rho(T)$ scales as $T^{4}$ in the low $T$ range and smoothly crosses over into a linear $T$ dependence at higher $T$. The dashed lines represent fits to the linear $T$ and $T^{4}$ dependencies, respectively. The inset shows the mobility $\mu_{0}$ at $T=2 \mathrm{~K}$ as a function of the density $n$. The gray line is the theoretically expected mobility due to short and long range impurity scattering.

At a given density $n$, each curve of $\Delta \rho$ shows a clear transition from a linear high temperature behavior $(\rho \sim T)$ to a superlinear $\left(\rho \sim T^{4}\right)$ behavior at low temperatures, as is expected from the BG model applied to electron-acoustic phonon scattering in graphene [8]. The crossover temperature between these two different regimes appears to be higher for higher carrier densities, in good accordance with the BG description presented above, where $\Theta_{\mathrm{BG}} \propto \sqrt{n}$.

We now quantitatively analyze our data in terms of the BG model. Considering the $e$-ph interaction as the major source of scattering, the temperature dependent resistivity of graphene can be obtained using the Boltzmann transport theory [8]:

$$
\Delta \rho(T)=\frac{8 D_{A}^{2} k_{F}}{e^{2} \rho_{m} v_{s} v_{F}^{2}} f_{s}\left(\Theta_{\mathrm{BG}} / T\right),
$$

where $D_{A}$ and $\rho_{m}$ are the acoustic deformation potential and the mass density of graphene, respectively, $v_{F}$ is the Fermi velocity, and the generalized BG function for graphene is given by the integration form $f_{s}(z)=$ $\int_{0}^{1} \frac{z x^{4} \sqrt{1-x^{2}} e^{z x}}{\left(e^{2 x}-1\right)^{2}} d x$. We remark that Eq. (1) is different from a typical BG formula for a 3D metal in three ways. First, the integrand contains $x^{4}$ instead of $x^{5}$, reflecting the 2D nature of electrons and acoustic phonons in graphene. Second, the relevant normalized temperature scale is $\Theta_{\mathrm{BG}}$ instead of $\Theta_{D}$, considering the fact $\Theta_{\mathrm{BG}}<\Theta_{D}$ in our experimental range. Third, the absence of backscattering for the carriers manifests itself in the factor $\sqrt{1-x^{2}}$ in the integrand, representing the chiral nature of the carriers in graphene [21].
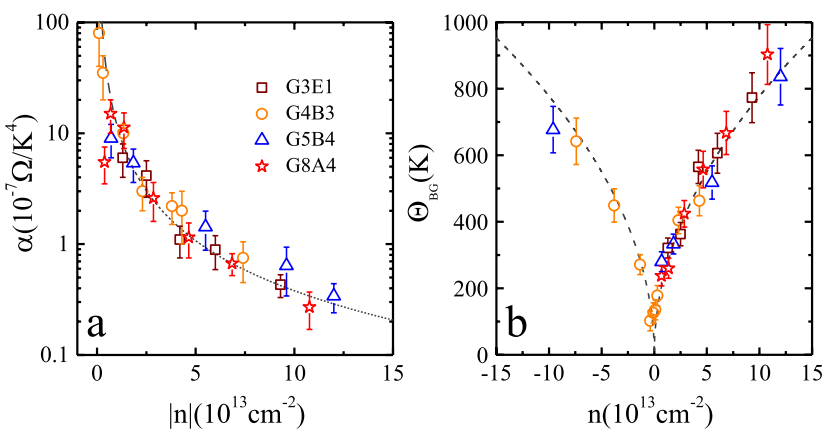

FIG. 3 (color online). (a) Scaling of the prefactors $\alpha(n)[\Delta \rho \approx$ $\alpha(n) T^{4}$ ]. Data points were obtained from $T^{4}$ fits of the $\rho(T)$ traces at different carrier densities and for different samples. The dashed line represents a theoretically predicted fit $\propto|n|^{-(3 / 2)}$. (b) $\Theta_{\mathrm{BG}}$ at different carrier densities [symbols are defined as in (a)]. The gray line is a fit to the theoretically predicted $\Theta_{\mathrm{BG}}=$ $2 \hbar v_{s} \sqrt{\pi n} / k_{B}$.

Taking the two opposite limits of the temperature ranges, Eq. (1) can be further approximated to $\Delta \rho \approx \gamma T$ for $T \geqslant \Theta_{\mathrm{BG}}$ and $\Delta \rho \approx \alpha T^{4}$ for $T \ll \Theta_{\mathrm{BG}}$, where the temperature independent proportionality coefficients are explicitly given by [8]

$$
\gamma=\frac{\pi D_{A}^{2} k_{B}}{4 e^{2} \hbar \rho_{m} v_{s}^{2} v_{F}^{2}}
$$

and

$$
\alpha=\frac{12 \zeta(4) D_{A}^{2} k_{B}^{4}}{e^{2} \hbar^{4} \rho_{m} v_{s}^{5} v_{F}^{2}}(\pi n)^{-(3 / 2)},
$$

where $\zeta$ is the Riemann-Zeta function.

Here we particularly note that $\alpha \propto|n|^{-3 / 2}$, while $\gamma$ is density independent. Using these properties, we obtain $\gamma$ and $\alpha(n)$ from the experimentally observed $\Delta \rho$ at fixed $n$. First, $\gamma \approx(0.14 \pm 0.01) \Omega / \mathrm{K}$ is estimated from the converging high temperature limit, scaling almost linearly down to $T \sim 0.2 \Theta_{\mathrm{BG}}$ (dotted line in Fig. 2(b), for example). This value is in reasonable agreement with the previous studies [9,10]. We then estimate $\alpha$ from each $\rho(T)$ curve at different densities by fitting to $\Delta \rho \sim T^{4}$ for the temperature range $T \leq 0.1 \Theta_{\mathrm{BG}}$. Figure 3(a) shows the resulting $\alpha$ versus $|n|$ in a wide range of experimentally accessible $|n|$ for four different samples. A clear trend of $\alpha(n) \sim|n|^{-3 / 2}$ can be seen [dashed trace in accordance with Eq. (3)].

The combination of the two coefficients $\alpha(n)$ and $\gamma$ allows us to compute the ratios of $D_{A}^{2} / v_{s}^{2}$ and $D_{A}^{2} / v_{s}^{5}$, respectively, and thus evaluate the values of $D_{A}$ and $v_{s}$ separately. Employing $\rho_{m}=7.6 \times 10^{-7} \mathrm{~kg} / \mathrm{m}^{2}$, and $v_{F}=10^{6} \mathrm{~m} / \mathrm{sec}$, we find that the average values for each parameter are $v_{s}=(2.6 \pm 0.4) \times 10^{4} \mathrm{~m} / \mathrm{sec}$ and $D_{A}=(25 \pm 5) \mathrm{eV}$, in good agreement with values reported in previous studies of suspended and substrate supported graphene devices, as well as for graphite and carbon nanotubes $[8,10,22-24]$. Using these we can now fit 


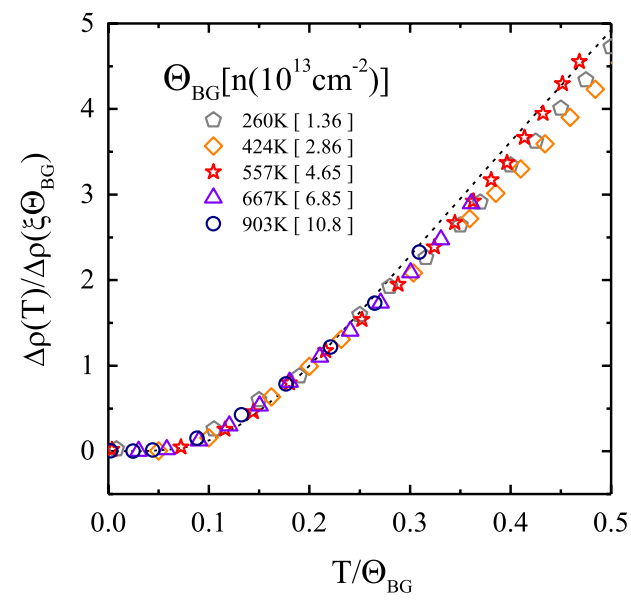

FIG. 4 (color online). Universal scaling of the normalized resistivity $\Delta \rho(T) / \Delta \rho\left(\xi \Theta_{\mathrm{BG}}\right)$ as a function of the normalized temperature $T / \Theta_{\mathrm{BG}}$, explicitly using the constant $\xi=0.2$. Data points correspond to $\Delta \rho(T)$ of sample G8A4 at different $n$ and $\Theta_{\mathrm{BG}}$ and are normalized with respect to $\Delta \rho\left(\xi \Theta_{\mathrm{BG}}\right)$ - the resistivity at $T=\xi \Theta_{\mathrm{BG}}$. The dashed line trace represents the theoretically predicted scaling of $f_{s}\left(\Theta_{\mathrm{BG}} / T\right) / f_{s}\left(\xi^{-1}\right)$ without the use of fitting parameters.

each experimental curve $\Delta \rho(T)$ by Eq. (1), using $\Theta_{\mathrm{BG}}$ as a single fitting parameter. Figure 3 (b) displays the experimentally determined $\Theta_{\mathrm{BG}}$ from this fit as a function of $n$ for both electrons and holes for all measured samples. The obtained $\Theta_{\mathrm{BG}}$ exhibit the predicted $\sqrt{|n|}$ dependence, explicitly demonstrating our capability to tune the BG temperature of up to $\sim 1200 \mathrm{~K}$ with the solid polymer electrolyte gate.

We finally discuss the universal scaling of $\Delta \rho(T)$. The experimental estimation of $\Theta_{\mathrm{BG}}$ now allows a direct test of the scaling behavior of $\Delta \rho(T)$, following Eq. (1), in all temperature ranges. Taking $T / \Theta_{\mathrm{BG}}$ as a dimensionless parameter, Eq. (1) can be rewritten in a dimensionless scaling form:

$$
\frac{\Delta \rho(T)}{\Delta \rho\left(\xi \Theta_{\mathrm{BG}}\right)}=\frac{f_{s}\left(\Theta_{\mathrm{BG}} / T\right)}{f_{s}\left(\xi^{-1}\right)},
$$

where $\xi$ is an arbitrary constant setting the normalization temperature relative to $\Theta_{\mathrm{BG}}$. Figure 4 displays the universal scaling behavior of Eq. (4) converted from Fig. 2. Here we simply choose $\xi=0.2$ to ensure $\Delta \rho\left(\xi \Theta_{\mathrm{BG}}\right)$ is within the experimentally accessible range. Remarkably, each normalized curve of $\Delta \rho$ with different $n$ (and thus different $\Theta_{\mathrm{BG}}$ ) falls on top of the theoretical curve, indicating the BG model of $e$-ph scattering fully explains $\rho(T)$ not only in the low and high temperature limits, but for all temperatures.
In conclusion, using the electrolyte we have achieved extremely high carrier densities of up to $|n|=$ $4 \times 10^{14} \mathrm{~cm}^{-2}$ in graphene samples. This advancement allowed us to observe a strictly 2D Bloch-Grüneisen behavior in the measured resistivity, exhibiting the linear $T$ to superlinear $T^{4}$ crossover, defined by the gate tunable characteristic temperature $\Theta_{\mathrm{BG}}$. Our quantitative analysis of the temperature dependent resistivity shows a universal scaling behavior of the normalized resistivity $\rho(T)$ with the normalized temperature $T / \Theta_{\mathrm{BG}}$, representing the $2 \mathrm{D}$ nature of the electrons and phonons along with the chiral nature of the carriers in graphene.

The authors thank E. H. Hwang, I. L. Aleiner, S. Das Sarma, and K. B. Efetov for helpful discussions, and K. F. Mak and S. Glinskis for sample preparation. This work is supported by the AFOSR0 MURI, FENA, and DARPA CERA. Sample preparation was supported by the DOE (DE-FG02-05ER46215).

[1] A. A. Abrikosov, Fundamentals of the Theory of Metals (North-Holland, Amsterdam, 1988).

[2] F. Bloch, Z. Phys. 59, 208 (1930).

[3] E. Grüneisen, Ann. Phys. (Leipzig) 408, 530 (1933).

[4] W. Meissner, Handbuch der Experimentalphysik XI/2 (Akad. Verlagsgesellschaft, Leipzig, 1935).

[5] H. L. Stormer et al., Phys. Rev. B 41, 1278 (1990).

[6] A. K. Geim and K.S. Novoselov, Nature Mater. 6, 183 (2007).

[7] A. K. Geim and P. Kim, Sci. Am. 298, 90 (2008).

[8] E. H. Hwang and S. Das Sarma, Phys. Rev. B 77, 115449 (2008).

[9] S. V. Morozov et al., Phys. Rev. Lett. 100, 016602 (2008).

[10] J. H. Chen et al., Nature Nanotech. 3, 206 (2008).

[11] E. V. Castro et al., arXiv:1008.2522.

[12] E. H. Hwang and S. Das Sarma, Phys. Rev. B 79, 165404 (2009).

[13] Matthew J. Panzer et al., Adv. Mater. 20, 3177 (2008).

[14] K. Ueno et al., Nature Mater. 7, 855 (2008).

[15] A. Das et al., Nature Nanotech. 3, 210 (2008).

[16] Kin Fai Mak et al., Phys. Rev. Lett. 102, 256405 (2009).

[17] J. Yan et al., Phys. Rev. B 80, 241417(R) (2009).

[18] In our experiment, $\rho_{0}$ is determined from the measured $\rho$ at the lowest temperature $(\approx 2 \mathrm{~K})$.

[19] S. Das Sarma, E. H. Hwang, and E. Rossi, Phys. Rev. B 81, 161407 (2010).

[20] For $|n| \lesssim 10^{13} \mathrm{~cm}^{-2}$, a strong activation behavior of $\rho(T)$ is observed for $T \geq 150 \mathrm{~K}$ as shown in $[9,10]$.

[21] E. H. Hwang (private communication).

[22] K. Bolotin et al., Phys. Rev. Lett. 101, 096802 (2008).

[23] L. Pietronero et al., Phys. Rev. B 22, 904 (1980); H. Suzuura and T. Ando, Phys. Rev. B 65, 235412 (2002).

[24] S. Ono and K. Sugihara, J. Phys. Soc. Jpn. 21, 861 (1966); K. Sugihara, Phys. Rev. B 28, 2157 (1983). 